\title{
Stoffübergang in Drehzylinderapparaten
}

\author{
Dr.-Ing. Alfred Schymalla
}

\section{Strömung in Drehzylinderapparaten}

Wer die Strömung in Drehzylinderapparaten das erste mal beobachtet, ist fasziniert.In dem Spalt zwischen dem drehbaren inneren Zylinder und der äußeren zylindrischen Wand kann man ein gleichmäßiges Wirbelmuster sehen, das über einen relativ weiten Drehzahlbereich stabil zu sein scheint. Dieses Wirbelmuster läßt sich verhältnismäßig leicht sichtbar machen und ist seit etwa 100 Jahren immer wieder Gegenstand zahlreicher Experimente und theoretischer Untersuchungen gewesen. Taylor hat als erster versucht, das Zustandekommen der nach ihm benannten Wirbel zu deuten (1). Weitere Autoren haben, aufbauend auf den Untersuchungen von Taylor, versucht, im Drehzylindersystem das Geheimnis der Turbulenz zu liiften.

In den letzten 10 Jahren hat die Verfahrenstechnik sich mit diesem interessanten Apparat immer wieder beschäftigt.

Das typische Wirbelmuster - die Taylerwirbel - zeigen sich dem Betrachter als Fischgrätenmuster (Bild 1), das durch die Überlagerung der Wirbelbewegung mit der Rotationsströmung ergibt. Die Wirbel sind ringförmig und parweise gegenläufig angeordnet.
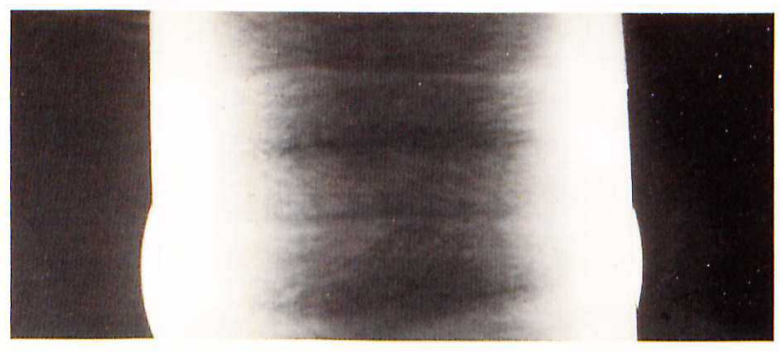

Bild 1: Wirbelströme im Drehzylinderapparat

Die Höhe der Wirbel entspricht nach Taylor der Spaltweite zwischen dem inneren und dem äußeren Zylinder. Untersuchungen des Autors ergaben, daß besonders bei zähen Flüssigkeiten die Wirbelhöhe das doppelte der Spaltweite uiberschreiten kann und das Wirbel verschiedener Höhe neberieinander existieren können.

Unter Verwendung der Spaltweite und der Drehzahl des inneren Zylinders läßt sich eine Reynoldszahl definieren, die meistens Taylorzahl genannt wird. Der Versuch, den Einfluß des Durchmessers des Innenzylinders bzw. des Durchmesserverhältnisses zu berüicksichtigen, hat zur Definition von mindestens 9 verschiedenen Taylorzahlen gefuihrt. Eine Taylorzahl, die alle Erscheinungen der Wirbelströmung ausreichend gut erfaßt, ist bisher noch nicht gefunden worden.

In Drehzylinderapparaten lassen sich wie bei der Rohrströmung die verschiedenen Strömungsarten - laminare, Wirbelströmung und Turbulenz - in Abhängigkeit von der Drehzahl erzeugen und bestimmten, charakte- ristischen Bereichen der Taylorzahlen zuordnen. Die laminare Strömung läßt sich nur sehr schwer beobachten und hat bisher sehr wenig Beachtung gefunden. Das größte Interesse haben die Taylowirbeln gefunden, die sich im Übergangsgebiet zwischen der laminaren und der turbulenten Strömung ausbilden. Sie sind leicht zu beobachten und gut reproduzierbar zu erzeugen. Die Strömung innerhalb der Taylorwirbeln ist laminar. Bei Steigerung der Drehzahl werden sie instabil. Diese Instabilität zeigt sich in einer Welligkeit der Wirbelgrenzen, die die Existenz einer zusätzlichen Wirbelbewegung innerhalb des Taylorwirbels anzeigt (Bild 2). Bei einer weiteren Steigerung der Drehzahl werden die Wirbelgrenzen immer unschärfer; der Übergang zur Turbulenz vollzieht sich für den Beobachter langsam.

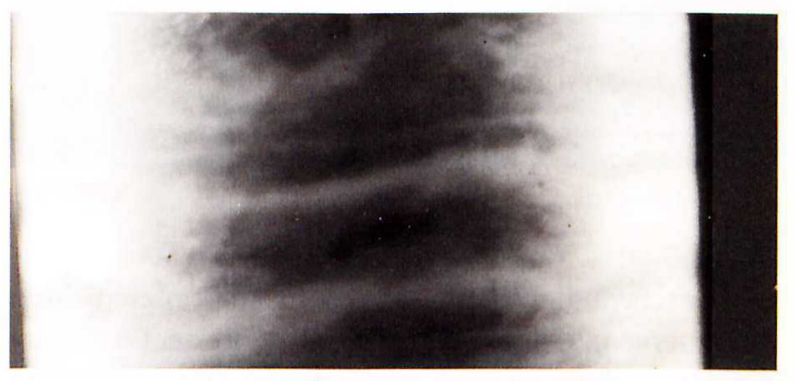

Bild 2: Wirbelströmung beim Übergang zur Turbulenz

\section{Dispersionsverhalten}

Die Geschwindigkeitsverteilung im Spalt eines Drehzylinderapparates zeigt Bild 3. Abgesehen von den gro-

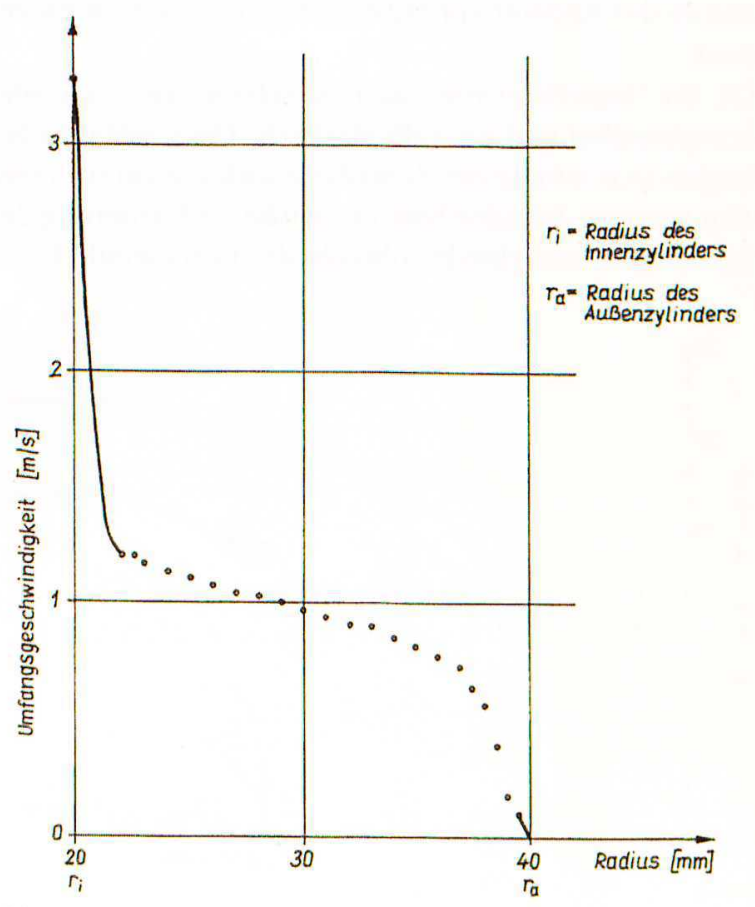

Bild 3: Geschwindigkeitsverteilung im Zylinderspalt 


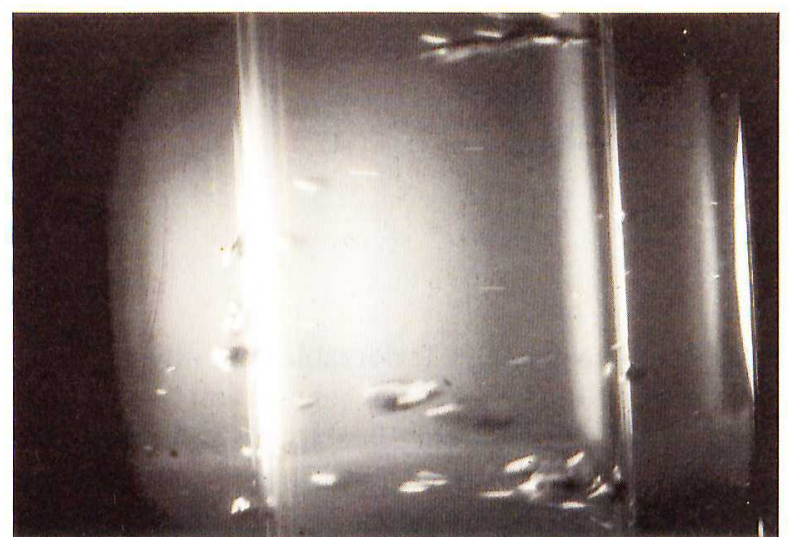

Bild 4: Blasenverteilung bei geringem Gasdurchsatz

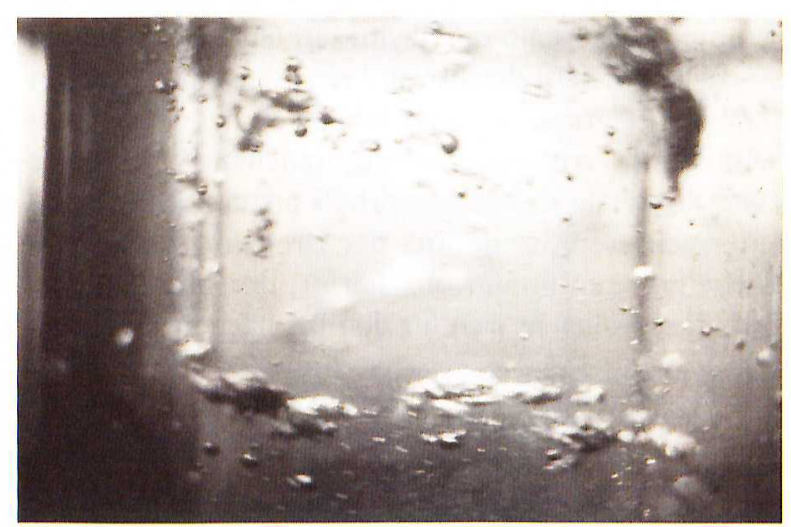

Bild 5: Blasenverteilung bei hohem Gasdurchsatz

ßen Geschwindigkeitsgradienten in den Grenzschichten des inneren und des äußeren Zylinders ist der Geschwindigkeitsgradient im Spalt relativ gering. Das miißte gute Voraussetzungen für die Dispergierung von Fliissigkeiten und Gasen schaffen.

Miteinander nicht mischbare Flïssigkeiten lassen sich im Drehzylinderapparat sehr einfach dispergieren. Die erreichbare Tropfengröße ist von der Apparategeometrie und der Drehzahl abhängig. Aus diesem Grunde wurde der Apparat zur Flüssig-Flüssigextraktion patentiert.

(2). Die Dispersion von Gasen ist schwieriger. Gase sind kompressibel und deshalb elastisch. Die Gasblasen bewegen sich infolge der Zentrifugalwirkung am rotierenden, inneren Zylinder. Eine merkliche Zerkleinerung der Blasen aber unterbleibt, obwohl der Geschwindigkeits-

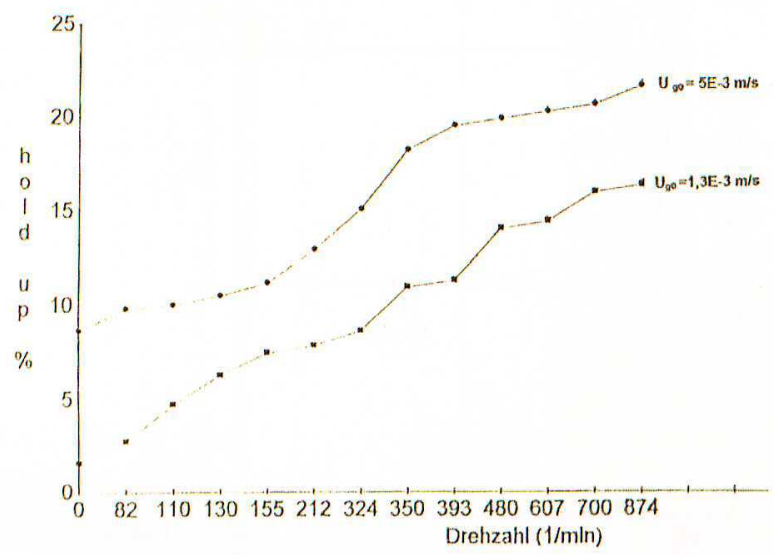

Bild 6: hld up als Funktion der Drehzahl gradient am rotierenden Zylinder am größten ist. Die Blasen werden infolge der Flüssigkeitsströmung in Bewegungsrichtung verformt (Bild 4, 5). Auffällig ist, daß die Blasen sich an den Grenzen der Taylorwirbel sammeln und dabei koaleszieren. Diese Koaleszenzneigung nimmt besonders bei höheren Gasgehalten mit steigender Drehzahl zu, wie Messungen des dispergierten Gasanteils (hold up) andeuten (Bild 6).

\section{Untersuchungen zum Stoffübergang}

Für die Messung des volumetrischen Stoffübergangskoeffizienten (kl a-Wert) wurde ein Laborapparat (Durchmesser $80 \mathrm{~mm}$, Höhe $500 \mathrm{~mm}$, Innenzylinder variabel) verwendet. In diesem Apparat wurde sauerstoffarmes Wasser im kontinuierlichen Betrieb im Gegenstrom mit Luft begast. Aus der Zunahme des Sauerstoffgehaltes und dem Wasserdurchsatz wurde der $\mathrm{kl}$ a-Wert errechnet. Die Darstellung der Abhängigkeit des kla-Wertes vom spezifischen Energieeintrag (Bild 7) im logarithmischen Maßstab ergibt Kurven, die im Bereich der laminaren Wirbel bis $\mathrm{P} / \mathrm{V}=20 \mathrm{~W} / \mathrm{m}^{3}$ einen Anstieg von etwa 0,2-0,25 aufweisen. Der Übergang zur Turbulenz ist bei $\mathrm{P} / \mathrm{V}=40 \mathrm{~W} / \mathrm{m}^{3}$ abgeschlossen und der Anstieg der Kurve beträgt $0,4-0,5$, wie es für Rührer häufig beobachtet wird.

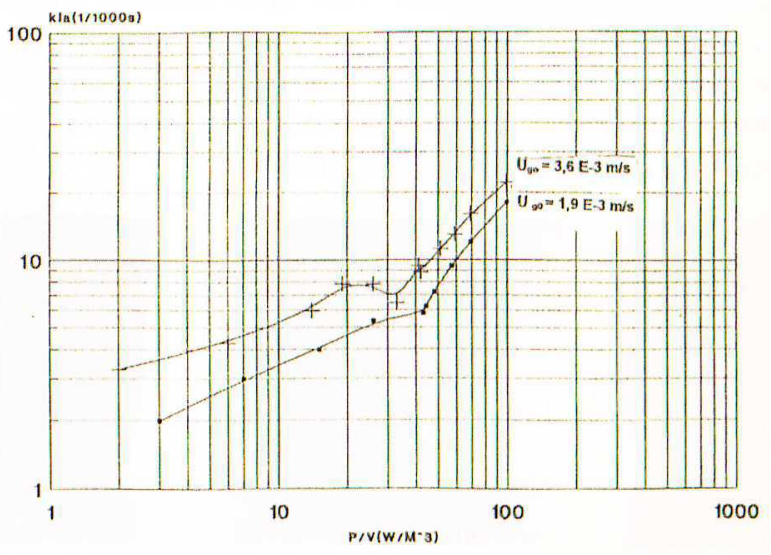

Bild 7: Stoffuibergang im System Wasser-Luft

Auch Messungen des Stoffübergangs in Abhängigkeit von der Gasleerrohrgeschwindigkeit ergeben für Rührreaktoren typischen Werte. Für Drehzylinderreaktoren ergibt sich noch ein zusätzlicher Einfluß durch die Fliissigkeitsleerrohrgeschwindigkeit.

Für das Stoffsystem Wasser / Luft wurde als empirische Abhängigkeit ermittelt :

$$
\begin{aligned}
\mathrm{kl} \mathrm{a}= & 0,05 \cdot(\mathrm{P} /)^{\mathrm{x}} \cdot \mathrm{U}_{\mathrm{GO}} \mathrm{y} \cdot \mathrm{U}_{\text {wo }}{ }^{\mathrm{z}} \\
\mathrm{x}= & 0,2-0,25 \text { laminare Wirbel } \\
\mathrm{x}= & 0,55 \text { turbulente Strömung mit Wirbelstruktur } \\
& \text { (enger Zylinderspalt) } \\
\mathrm{x}= & 0,4-0,5 \text { turbulente Strömung ohne Wirbel- } \\
& \text { struktur (weiter Zylinderspalt) } \\
\mathrm{y}= & 0,4-0,6 \text { in Abhängigkeit von der Koaleszenz } \\
\mathrm{z}= & 0,5
\end{aligned}
$$




\section{Anwendung von Drehzylinderapparaten}

Das interessante Wirbelmuster in Drehzylinderapparaten hat zu einer Reihe von Versuchen und technischen Anwendungen geführt :

- Fliissig-Fliissigextraktion

- Destillation

- dynamische Mikrofiltration

- Flockulation

- Bioreaktor

- Synthesen in Mehrphasensystemen

- Gaswascher

Füir den Reaktionstechniker sind besonders die Taylorwirbel von Interesse; jeder Taylorwirbel kann als eine Ruihrkesselstufe aufgefaßt werden. Krause (3) hat das Verweilzeitverhalten von Drehzylinderapparaten im Einund Zweiphasenbetrieb (Gas- und Fluissigphase) untersucht :

- Im Einphasenbetrieb entspricht die gemessene Rührstufenzahl etwa der Anzahl der Taylorwirbel, solange diese eine laminare Struktur haben. Die Verweilzeitverteilung bleibt auch noch günstig,wenn die Laminarität der Wirbel verlorengeht.

- Im Gas-Fluissigbetrieb ist die Verweilzeitverteilung nur in einem engen Drehzahlbereich günstig. Der Zerfall der Taylorwirbel wird zu höheren Drehzahlen verschoben. Im Bereich der turbulenten Strömung werden nur wenige Ruihrstufen pro Meter Reaktorhöhe erreicht.

In Drehzylinderapparaten von technisch relevanter Größe werden die kritischen Reynoldszahlen (Taylorzahlen) für den Zerfall der Taylorwirbel und für die Ausbildung der Turbulenz schon bei geringen Drehzahlen iiberschritten. Die günstige Verweilzeitverteilung der Wirbelströmung läßt sich damit nur in Apparaten im kleintechnischen Maßstab nutzen nutzen.

Aus diesem Grunde wird der Drehzylinderapparat wahrscheinlich immer nur ein interessanter Laborreaktor mit Optionen auf die kleintonnagige Produktion bleiben.

\section{Literatur}

(1) Taylor G. I., Phil.Trans. R. Soc. 1923 A 223,289-343

(2) Maycock, US-Patent 1945

(3) Krause, B.; Dissertation 1988

\section{Verfasser}

Dr.-Ing. Alfred Schymalla

Wasser- und Entsorgungstechnik

im TGZ Wildau

Bahnhofstr. 1

Tel.: (03375) 508385 